This article is licensed under the Creative Commons Attribution-NonCommercial 4.0 International License (CC BY-NC) (http://www.karger.com/Services/OpenAccessLicense). Usage and distribution for commercial purposes requires written permission.

\title{
High Rate of Early Posterior Capsule Opacification following Femtosecond Laser-Assisted Cataract Surgery
}

\author{
Benjamin Rostami $^{\mathrm{a}} \quad$ Jack Tian $^{\mathrm{a}}$ Nicholas Jackson ${ }^{\mathrm{b}}$ Rustum Karanjia ${ }^{\mathrm{a}, \mathrm{c}, \mathrm{d}}$ \\ Kenneth $\mathrm{Lu}^{\mathrm{a}, \mathrm{c}}$ \\ a Doheny Eye Center, Department of Ophthalmology, David Geffen School of Medicine at \\ UCLA, Los Angeles, Calif., USA; ${ }^{b}$ Division of General Internal Medicine and Health Services \\ Research, Department of Medicine Statistics Core, David Geffen School of Medicine at \\ UCLA, Los Angeles, Calif., USA; 'Doheny Eye Institute, Los Angeles, Calif., USA;

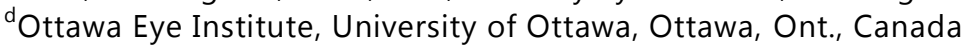

\section{Keywords}

Cataract · Posterior capsule opacification - Femtosecond laser-assisted cataract surgery

\begin{abstract}
Purpose: To compare the rates of rapid posterior capsule opacification (PCO) formation in the first 3 months following femtosecond laser-assisted cataract surgery (FLACS) to manual anterior capsulorhexis. Methods: Retrospective review of 29 cases of FLACS, comparing the rates of PCO in the first 3 months following surgery to 50 consecutive cases of manual anterior capsulorhexis. Results: Seven of the 29 FLACS cases developed PCO requiring capsulotomy at 3 months, while none of the control cases required a capsulotomy over the same time period $(p<0.05)$. Conclusion: There is an increased incidence of early-onset PCO following the use of femtosecond laser in cataract surgery that is otherwise unfounded in man-
\end{abstract}




\section{Case Reports in Ophthalmology}

Case Rep Ophthalmol 2016:7:213-217

(c) 2016 The Author(s). Published by S. Karger AG, Base www.karger.com/cop

Rostami et al.: High Rate of Early Posterior Capsule Opacification following Femtosecond Laser-Assisted Cataract Surgery

ual capsulorhexis. This suggests that the use of a femtosecond laser could increase the risk of this novel postoperative complication.

(C) 2016 The Author(s)

Published by S. Karger AG, Basel

\section{Introduction}

Femtosecond laser-assisted cataract surgery (FLACS) is a relatively recent evolution of traditional manual phacoemulsification cataract surgery. The laser can be used to perform the capsulotomy, lens fragmentation, and corneal incision, which results in enhanced precision, decreased phacoemulsification power, and improved centration of the intraocular lens (IOL) [1, 2]. This, in turn, should result in better vision and long-term outcomes [3]. Posterior capsule opacification (PCO) is a recognized complication of cataract surgery [4]. Though common, PCO usually presents several months to years after cataract surgery. In this study, we sought to report and investigate the incidence of rapid PCO formation in the first 3 months following the use of FLACS. Furthermore, we examined the efficacy of anterior capsule polishing in reducing the rate of early-onset PCO formation in FLACS.

\section{Methods}

A retrospective chart review was completed of 29 consecutive cases of FLACS (capsulotomy and laser fragmentation) and 50 consecutive cases of cataract surgery with manual anterior capsulorhexis and standard phacofragmentation (control), which were performed over the same time period by the same surgeon at the Doheny Eye Center, UCLA. The same LENSAR cataract laser was used for all FLACS cases with the same parameters for 5.0-mm capsulotomy and lens fragmentation. All patients had the AMO Z9002 3-piece Tecnis IOL. The two groups were compared in terms of the incidence of rapidly forming, visually significant PCOs that required Nd:YAG laser capsulotomy within 3 months of the initial cataract surgery. Further, the FLACS cases were divided based on the use of anterior capsule polishing in the surgery protocol and compared to evaluate the efficacy of this technique in reducing the rate of PCO formation. Analysis was done with Fisher's exact test, and conducted in Stat version 13 (StataCorp LP, College Station, Tex., USA).

\section{Results}

The study evaluated 79 eyes from 63 patients. The mean age was $72.3 \pm 10.9$ years in the control group, and $72.6 \pm 5.5$ years in the FLACS group. The groups had similar male-tofemale ratios, and overall, there were no significant differences in the demographics between the control and experimental groups (table 1).

There were no cases of PCO recorded within the first 3 months in any of the 50 control cases of cataract surgery with manual anterior capsulorhexis and standard phacofragmentation. In contrast, seven of the 29 FLACS cases developed PCO requiring capsulotomy over the same time period $(\mathrm{p}<0.05)$. Anterior capsule polishing was not found to make a significant 


\section{Case Reports in Ophthalmology}

Case Rep Ophthalmol 2016;7:213-217

DOI: $10.1159 / 000449124$

C 2016 The Author(s). Published by S. Karger AG, Basel
www.karger.com/cop

Rostami et al.: High Rate of Early Posterior Capsule Opacification following Femtosecond Laser-Assisted Cataract Surgery

difference in the rate of PCO formation ( $\mathrm{n}=12$ FLACS without polish; 17 FLACS with polish; $\mathrm{p}=0.769$ ) (table 2, table 3). Additionally, it was noted in the procedural notes from Nd:YAG capsulotomy that the PCOs were of a fibrotic type that was difficult to laser.

\section{Discussion}

In this study, we sought to investigate the incidence of a unique new postoperative complication of FLACS, namely the rapid formation of fibrotic PCO in the first 3 months following the procedure. We found a statistically significant difference in the rate of this type of PCO when comparing cases of FLACS to manual anterior capsulorhexis and standard phacofragmentation.

Our small retrospective series suggests that the frequency and nature of PCO may be different and worth noting in FLACS. Despite previous studies that demonstrated the laser's relatively safe use, many experienced surgeons remain apprehensive to the adoption of the laser into their protocol, expressing concerns of a learning curve and possible unforeseen complications associated with the laser $[5,6]$. In this series, we describe one of those unanticipated outcomes.

PCO was traditionally known to be a natural consequence of cataract surgery, the socalled 'secondary cataract' or 'after-cataract' that forms as displaced epithelial cells migrate posteriorly, make their way behind the IOL, and take residence on the posterior capsule [4]. As such, one proposal for the mitigation of rapidly forming PCO as a result of FLACS is anterior capsule polishing. However, the efficacy of this additional step in the procedure did not affect the rates of PCO in the FLACS group, and is still debated in the literature [7]. While polishing the anterior capsule is not a universally accepted way to delay the formation of PCO, in our situation, due to the alarming rate of early PCO formation, the surgeon decided to add this otherwise easy, straightforward step to try to address the issue at hand. Our small sample did not show a statistically significant lower rate of PCO with anterior capsule polishing, which could be because anterior lens epithelial cells might not be the ones responsible for the formation of the PCO.

The etiology of this reported phenomenon could stem from the previously described 'learning curve' $[5,6]$. While all surgeons experience a learning curve when adapting their surgical technique to including new technology like FLACS, the rates in the control group by the same surgeon demonstrate the surgeon's skill and experience of having performed over 6,000 cataract surgeries. Additionally, in our study, the incidence of PCO was evenly distributed throughout rather than heavily concentrated towards the beginning of the time frame, which would have been more indicative of the presence of said learning curve. However, investigation as to the presence of a learning curve would require further long-term observation of PCO formation, which we hope to do and present at a later time. Alternatively, rapid PCO formation may be a result of thermomechanical damage to the lens epithelial cells, as the effects of thermomechanical damage to other ocular membranes have previously been described [2]. Of note, there was no association between PCO formation and the presence of other underlying chronic medical illnesses such as diabetes mellitus which could cause ocular damage. Four of the patients in our cohort were noted to have diabetes, none of whom were among those who had rapid PCO formation. 
The high rate of PCO formation within 3 months suggests that there is a fundamental difference between the PCO formed in FLACS, which was also evidenced by the fibrous nature of the PCO, which required higher Nd:YAG energy levels to disrupt. Understanding the differences accounting for this increased fibrotic nature of the PCO could lead to improved techniques to mitigate the formation of PCO post-FLACS.

In the future, we would like to continue to investigate the observed phenomenon using a more robust prospective controlled trial. Furthermore, it might be worthwhile to compare the incidence of such outcomes amongst the various lasers that are on the market, which was difficult to do so at the small center where our pilot study was based. Since this study was conducted, we have already begun to collect data on two other lasers and hope to be able to present our findings at a later time, once we have a better sense of how the different devices on the US market compare both with respect to rapid PCO formation, as well as other phenomena such as intraoperative miosis.

\section{Statement of Ethics}

Informed consent was obtained. Institutional Review Board approval was obtained and the study protocol was approved by the institute's committee on research and was seen to meet institutional standards.

\section{Disclosure Statement}

All authors report no financial disclosures or potential conflicts of interest. This work was supported by CTSI grant UL1TR000124UCLA (Clinical and Translational Science Institute).

\section{References}

1 Packer M, Teuma EV, Glasser A, Bott S: Defining the ideal femtosecond laser capsulotomy. Br J Ophthalmol 2015;99:1137-1142.

-2 Palanker DV, Blumenkranz MS, Andersen D, et al: Femtosecond laser-assisted cataract surgery with integrated optical coherence tomography. Sci Transl Med 2010;2:58ra85.

-3 Kovács I, Kránitz K, Sándor GL, et al: The effect of femtosecond laser capsulotomy on the development of posterior capsule opacification. J Refract Surg 2014;30:154-158.

-4 Vasavada AR, Raj SM, Shah GD, Nanavaty MA: Posterior capsule opacification after lens implantation: incidence, risk factors and management. Expert Rev Ophthalmol 2013;8:141-149.

5 Abell RG, Darian-Smith E, Kan JB, Allen PL, Ewe SY, Vote BJ: Femtosecond laser-assisted cataract surgery versus standard phacoemulsification cataract surgery: outcomes and safety in more than 4000 cases at a single center. J Cataract Refract Surg 2015;41:47-52.

-6 Roberts TV, Sutton G, Lawless MA, Jindal-Bali S, Hodge C: Capsular block syndrome associated with femtosecond laser-assisted cataract surgery. J Cataract Refract Surg 2011;37:2068-2070.

7 Menpace R, Wirititsch M, Findl O, Buehl W, Kriechbaum K, Sacu S: Effect of anterior capsule polishing on posterior capsule opacification and neodymium:YAG capsulotomy rates: three-year randomized trial. J Cataract Refract Surg 2005;31:2067-2075. 
Table 1. Demographics by group

\begin{tabular}{llll}
\hline & Control & FLACS with polishing & FLACS without polishing \\
\hline Average age, years & $72.3 \pm 10.9$ & $74.0 \pm 6.8$ & $71.1 \pm 5.1$ \\
$\%$ Male & $63 \%$ & $50 \%$ & $57 \%$ \\
$\%$ Female & $37 \%$ & $50 \%$ & $43 \%$ \\
\hline
\end{tabular}

Table 2. Incidence of PCO and Nd:YAG capsulotomy at 3 months following surgery

\begin{tabular}{|c|c|c|c|c|c|}
\hline Group & Sample size, $\mathrm{n}$ & PCO & Proportion & Nd:YAG capsulotomy & Proportion \\
\hline Manual anterior capsulorhexis (control) & 50 & 0 & $0 \%$ & 0 & $0 \%$ \\
\hline FLACS with polishing & 17 & 3 & $18 \%$ & 2 & $12 \%$ \\
\hline FLACS without polishing & 12 & 4 & $33 \%$ & 3 & $25 \%$ \\
\hline
\end{tabular}

Table 3. Comparison of groups

\begin{tabular}{llll}
\hline Outcome & FLACS vs. control & FLACS + polish vs. control & FLACS vs. FLACS + polish \\
\hline PCO & $\mathrm{p}=0.016$ & $\mathrm{p}=0.041$ & $\mathrm{p}=0.769$ \\
Nd:YAG capsulotomy & $\mathrm{p}=0.029$ & $\mathrm{p}=0.180$ & $\mathrm{p}=0.657$ \\
\hline
\end{tabular}

